\title{
Analysis on the Communication Strategy of Xi'an City Image in the New Media Environment
}

\author{
Minjing Wang ${ }^{1, *}$
}

\author{
${ }^{1}$ Xi'an FanYi University, Xi'an, Shaanxi, China \\ *Corresponding author. Email: angelina1224@126.com
}

\begin{abstract}
In the new media environment, the media's communication subject, communication channels, and communication methods have all changed. In the process of building and disseminating the image of the city, Xi'an should change the previous top-down propaganda concept, make full use of the advantages of new media, enrich the communication subject, give play to the interactive features of new media, and realize cross-media communication strategy under the guidance of the overall communication concept. These can make the image of Xi'an, a historic city and "capital of hard technology", be known and recognized by the visitors, so as to better enhance the city's outward orientation and reputation.
\end{abstract}

Keywords: new media, "capital of hard technology", city image, communication strategy

\section{INTRODUCTION}

The emergence of digital technology has changed the urban lifestyle, living space, the relationships between people and between human and the environment. The increasing complexity of modern society makes people's actual range of action, energy and attention limited, and it is impossible to maintain empirical contact with the entire external environment related to them. They can only get used to understanding their environment through various media organizations. People live in what Lippmann called the "pseudoenvironment", so the urban environment constructed by the media has become an important window for the public to recognize the city, and it has also become the main channel for people to investigate the construction of the city's image.

\section{THE INFLUENCE OF NEW MEDIA ON THE COMMUNICATION OF CITY IMAGE}

With the development of Internet technology, the forms and contents of new media have become more diverse. With the advent of the web3.0 era, various media are developing in the direction of mutual integration, and more precise, intelligent and segmented content push will make the

*Project: This article is a phased research result of the Shaanxi Provincial Department of Education's 2020 Special Scientific Research Project "Media Communication Strategies in Shaping the Image of Xi'an 'Capital of Hard Technology'" (Project No." 20JK0177) dissemination of information more effective. The development of new media on society cannot be underestimated, so many cities have begun to face up to the influence of new media. According to the 45th China Internet network development statistics, in 2019, Baidu's mobile government service search volume was 20.197 billion times, the number of Weibo accounts of government agencies certified by the Sina platform was 139,000 , the number of government affairs headlines established by governments at all levels was 82,937, and government affairs Douyin accounts are 17,380 (as of December 2019). [1] These officially established WeChat and Weibo platforms or media platforms such as government affairs headlines, Douyin and other media platforms provide services or publish information, interact with local residents or foreign tourists, shorten the distance between the government and the people, and improve the image of the city. The "decentralization" of new media has made the public's evaluation channels smoother. These public opinions active on new media platforms play a very important role in spreading the positive or negative image of the city.

Accompanying the development of new media is that the main body, content, and methods of communication have undergone profound changes. Different media strategies will have completely different communication effects. Therefore, it is a must to face up to the changes brought about by media changes. For every city, the purpose of communication is the same, hoping to enhance or 
strengthen the competitiveness of the city in the country and the world through the communication of the city image, occupy a certain competitive advantage, and then contribute to the city's infrastructure and economic development, providing a more favorable and powerful driving force for the improvement of local quality of life.

As the ancient capital of the thirteen dynasties, Xi'an took the lead in raising the banner of "hard technology" in 2017. The "hard technology" mentioned here refers to high-precision technology represented by aerospace, photonic chips, new materials, genetic technology, brain science, artificial intelligence, etc. It has a high threshold and is difficult to be copied and imitated. It requires five to more than ten years of accumulation to form. This concept was first proposed by Mi Lei, $\mathrm{PhD}$ of Optics, Xi'an Institute of Optics and Mechanics, Chinese Academy of Sciences. Subsequently, the term "hard technology" gradually became familiar to the public. In 2017, Xi'an clearly proposed to build a "capital of hard technology" and implemented a series of development strategies such as "hard technology + +, "Internet +", "university +" and "entrepreneurship platform +". Conceptually speaking, the enrichment of the connotation of the city's image, whether it is a proactive communication behavior or a communication behavior driven by passive competitive pressure, and whether the purpose of communication is to attract investment, promote tourism, establish city logos and values, or enhance local residents' sense of identity and belonging, it is undeniable that these concepts of city image dissemination will inevitably be deeply influenced by new global dissemination forces. The number and type of new media are constantly being introduced. In the continuous game and integration with traditional media, the ecological environment of the media has been changed, all links of communication practice activities have been changed, the past information dissemination methods have been subverted, and people's life style has even been changed. So, in the era of new media, the diffusion mode and path of urban image communication have undergone great changes. Therefore, it is necessary to consider the city image of Xi'an as the "capital of hard technology" in the context of new media.

\section{ANALYSIS ON THE STATUS QUO OF CITY IMAGE COMMUNICATION IN XI'AN}

In the process of shaping the image of Xi'an as the "capital of hard technology", the positioning of the city determines the "hard content" and general direction of the city's image communication. "The symbolic reality displayed by the media using various symbols, replacing the objective social reality, has become the main source of cognition for people to shape their image. In this media-based society, the image of the city cannot be separated from the construction of news media. The shaping and formation of the image of the city is closely related to the reporting activities of the mass media." [2] In the new media environment, abundant network resources provide good conditions for the spread and shaping of the image of Xi'an "capital of hard technology", but there are also certain problems.

\section{A. The information source is single, and the update is not instant enough}

At present, the promotion of large-scale outdoor media, television, newspapers, magazines and other traditional media types is the key to the promotion of Xi'an's "capital of hard technology". For example, in Xi'an High-tech Zone, people can often see words such as "hard technology leading highquality development" on outdoor displays. On the Internet, SNS social media such as Sina Weibo on government affairs microblogs such as "published in Xi'an", "in Xi'an", WeChat public account "published in Xi'an", "Xi'an hard technology capital"... search engines and online audio-visual media have become the main channel of information dissemination and diffusion in the communication of city image. According to their own characteristics, different media types follow their own communication laws in various forms in the communication process. Communication symbols such as images, words, and sounds complement each other and bring out the best in each other. Although using the advantages of new media, the channels for information dissemination are diversified but the effect is average. First, the information source of the dissemination is mostly government-level related parts, and the information source is single. Second, the information update is not timely and the communication effect is not good. Taking the WeChat public account of "Xi'an, capital of hard technology" as an example, the content is mainly hard technology bulletin, which is similar to the government work briefing. It lacks new ideas in form and the update time stopped in December 2018. As the Shaanxi news portal website, Westwest.com has a hard science and technology column on the homepage, and occasionally releases "hard science and technology" related news reports, but the news sources are more traditional media. The thematic section on the right side of the webpage, so far, the publicity report on the Global Hard Technology Conference has stayed in 2018. From this, it is not difficult to see that the timeliness of the new media has not played its due immediacy. 


\section{B. The interactivity of information dissemination is weak}

Even in the context of new media, traditional mass media still continues the communication logic of vertical discourse power. As the main theme of city image communication, government departments have accumulated a lot of experience in the use of traditional mass media, and they are also used to carrying out most of the activities of city image communication through traditional media. Therefore, the government-level policy agenda is the starting point of communication, and it is widely disseminated to the public through mass media. The communication of the image of the "capital of hard technology" is more common in traditional media and government media. Generally, the content of communication is highly related to policies or technological developments. In the process of making an impact on the agenda, the policy agenda affects the media agenda. Although the personal agenda is added due to the addition of new media, the effect is minimal. During the "2020 Global Hard Technology Development Conference" that demonstrates the strength of hard technology, there is often a small climax in the annual hard technology related reports. Sina Weibo, as a social media, showed that the attention to the capital of hard technology rose to a small climax in September 2020, with 4.493 million views and only 2,254 discussions. The number of readings and discussions in the rest of the time tends to zero. It is not difficult to find by analyzing the \#2020 全球硬科技会议（\#2020 Global Hard Technology Development Conference\#) topic list that traditional media accounted for more than $50 \%$ of the reports of this media event. Although there are 291,000 reads, the total number of reposts is only 28 , the number of comments is 6 , and the number of likes is 13 . Of the 6 comments, only 2 are visible, and the rest are invisible. To set the evaluation criteria of the use effect in urban image communication based on the current social needs, it should look at whether the media has established a platform for "dialogue" with the target audience. The generation and smooth progress of the dialogue is the basis for achieving the next goal. The current communication often focuses on "communication" itself rather than "dialogue."

\section{The media choice is single, and the cross-media communication is weak}

The characteristics of the media itself inevitably have certain limitations and deficiencies, and different public channels for receiving information are not the same, and there is a certain degree of remoteness. Therefore, the communication channels for the image of the city should be diversified. In view of the current situation where most of the public are inadvertently exposed to the spread of the city's image, the communicators can enhance the layout of the environment, through outdoor media, traffic media, and broadcast media to achieve multi-channel and multi-screen delivery, and combine the characteristics of the media to form creative content with unique patterns and create a city image information contact, experience and easy-to-accept atmosphere. In response to the hot topics of public concern, the creation of the topic should be strengthened, and the creative expression of the city image should be strengthened for the problem that the public is less reposted and recommended, so as to promote the formation of hot spots in the city image communication in social media, and expand the breadth of the city image communication, as well as to attract more people to participate and stimulate their participation and spread enthusiasm. In the process of shaping and disseminating the image of Xi'an "capital of hard technology", this combination of media and crossmedia communication is relatively weak. In 2020, People's Video and 2020 Global Hard Technology officially launched the "Hard Technology by My Side" short video solicitation event. The event was announced on Weibo. However, channels such as search engines and major short video apps have launched corresponding reports and publicity, and their influence is minimal. The Global Hard Technology Innovation Conference has been set up in Xi'an for four consecutive years since 2017. As a technological feast, this is an excellent opportunity for Xi'an to spread the new image of the city. The planning of this event is also quite creative, such as cross-media and multi-channel promotion, topic interaction on short video platforms such as Kuaishou or Douyin, to expand the visibility of the event and its influence. Supplemented by careful planning by means of prize consultation, etc., it is believed that it will encourage more people to participate in it, take the initiative to learn about hard technology, experience the application of hard technology in life, and leave a clear impression on the 2020 Global Hard Technology Conference and even Xi'an as the "capital of hard technology".

\section{THE OPTIMIZED STRATEGY OF CITY IMAGE COMMUNICATION}

The continuous innovation of new media technology has triggered changes in the media ecological environment and formed new communication trends. It has also affected all the links of content production, creative expression, media selection, information output, and effect evaluation of the image dissemination of Xi'an "capital of hard technology". Therefore, the image of the "capital of hard technology" must follow the laws of communication, grasp new trends, and explore new communication strategies. 


\section{A. Participation of multiple subjects, and enriching the communication perspective and content}

Psychologist William Stephenson believes that instead of treating the medium as a tool, it is better to treat it as a toy. People play with the medium for the purpose of games rather than out of utilitarian considerations. The shaping of the image of the "capital of hard technology" news reports are related to the "instrumental" of the media, while non-news reports are related to the "games" of the media. As far as the communication and promotion of the city image is concerned, it is necessary to change the concept and model that the government alone takes the lead, and transform it into a mode of communication activities involving the government, media, enterprises, and the public. It is also necessary to make every effort to use new media forms to continuously enrich the perspective and content of city image communication, and promote the normalization of city image communication. City image communication is the construction of identity between self and others. Therefore, urban image communication must fully recognize the identity and antagonism of the identity and self-identity of others, and the contradiction between constructivity and deconstruction, and make full use of "self-shaping" and "other shaping", "shaping" and "correction", and positive and positive communication efficiency released by the game and collusion between "autobiography" and "biography". [3] As far as the "self-shaping" of the media is concerned, it is necessary to strengthen the promotion and publicity of the image of the city, and strengthen the creation of intentional topics especially in the planning of some urban events in accordance with the characteristics of hot topics of public concern. For example, the unabated Xi'an "Air Show" often becomes an excellent opportunity for "black technology" and "hard technology" to show up. The holding of "Xi'an Marathon" and some large-scale sports events has also become a platform for Xi'an to demonstrate its "science and technology" strength. In addition, it is also a must to play the role of "other shaping" and use other media platforms for promotion. For example, the two sessions of "Xi'an Marathon" are also full of "sense of science and technology", from the face recognition technology on the site to the first Beidou Xi'an Marathon race pass to the 4D medals that will be illuminated, and to the full integration of 5G technology for the first time in 2019. For the first time in the province, runners and on-site spectators of 5G+MR mixed reality applications can stand in front of $5 \mathrm{G}+$ cloud cameras and take photos with the beautiful scenery along Xi'an Marathon. Daming Palace National Heritage Park Danfengmen Square, a 5G experience hall of more than 1,000 square meters, will allow contestants to experience the magical charm of technology at close range. As a sporting event, with the help of "technology", Xi'an Marathon has not only greatly improved the service efficiency of the event, provided convenience to the majority of runners, but also gained widespread praise from the outside world. In the process of zero-distance contact between the on-site runners and the masses of science and technology, many people have taken videos and uploaded them to social media, which strengthened the identification of "others". This is undoubtedly a very good example of the city image.

\section{B. Taking audiences as priority, and using the help of new technologies to innovate communication methods}

There is a close relationship between the formation of a city image and its communication, media issues and reporting framework, but it is by no means a simple causal relationship. It is closely related to the values and expectations of the audiences as the cognitive subject, as well as the city's political economy and culture. Therefore, the audience-oriented concept must be established in the process of communication. The audiences' information reception into preferences and habits has become a very important criterion for measuring the effectiveness of communication. "The core of the future media competitiveness is data. Statistical analysis through those dynamic data, including structured and unstructured data, can reveal the trajectory of user behavior and emotional needs, and obtain more accurate user interest trends, personalized needs, and new business development trends and other important information." For the promotion and publicity of the city's image, these data are no longer cold numbers, but primary information and resources indispensable for content production and marketing. Through them, the segmentation of the audience and the push of personalized information can be realized. Cities should listen to the needs of the people with an attitude of equality and sharing, and value the audience's experience. Now that $5 \mathrm{G}$ commercialization is in full swing, "If $4 \mathrm{G}$ has changed the way of communication between people, then the $5 \mathrm{G}$ era will deepen the relationship between the media and society, and accelerate the intelligentization and autonomy of society. This will be a truly convergent network era." [4] The advent of the $5 \mathrm{G}$ era makes the communication of the city image more intuitive and immersive, paying more attention to the interaction and sense of experience in the communication process, and at the same time giving the people more discourse, making people have more initiative in the process of building the city image. Therefore, in the new media environment, it is more important to be audience-oriented and establish a benign information feedback mechanism. 


\section{Conforming to the development of the times and realizing cross-media communication}

Facts have proved that the needs of different individuals and organizations in the process of receiving city image and dissemination information are very different, and the media are also different. Therefore, in order to achieve effective communication, it is necessary to adapt to the changes and development trends of the current communication environment and achieve crossmedia communication. The first is to use big data to refine and differentiate the target groups. On this basis, the communication subject, strategy, text and other content can be set according to different communication goals; finally, according to the audience's media acceptance habits, the method of "differentiated communication" can be adopted to achieve accurate information dissemination, to deliver the most suitable information to the target audience, and to improve the effectiveness of communication. The era of big data provides people with convenient access to media contact behaviors and preferences of the audience. The advantages of new media interaction and immediacy have laid a good foundation for the city to achieve "differentiation", "individualization" and "precision" communication. The era promotes the spread of the image of the city between people to a new level of "communication" and even "dialogue". [5] In the process of city image communication, it should first be clear that the relationship between traditional media and new media is not a competition and opposition. Therefore, as far as the media itself is concerned, it should follow the new trend of media integration, strengthen cooperation with new media, and use the convenient and efficient promotion platform of new media to form a synergy in shaping the city image. On the other hand, the media should make full use of the communication advantages of various media forms to achieve the best communication effect with the appropriate content product portfolio. Under the guidance of the integrated communication concept, these unified information can repeatedly stimulate the public's audio-visual system, and deepen the impression and evaluation of the distinctive personality of the city image.

\section{CONCLUSION}

The communication of city image is the active proliferation of the active will of city function positioning and the integrated communication of city image. The relationship between Xi'an's historical civilization and modern technology is not in conflict, and the image of the "Ancient City of Xi'an" and the city image of "capital of hard technology" are not contradictory. In the era of new media, how to complement each other's strengths and better publicize and promote Xi'an is the core issue. New media and methods of communication that are constantly emerging in the current communication environment should be highly sensitive in shaping the image of Xi'an "capital of hard technology". It is necessary to constantly try, combine and update their own communication methods. It is also necessary to exploit the potential of all parties in the image of the city, closely link the overall communication goal with the communication power, make technology better serve the communication activities, and strengthen the effectiveness of communication in the diversified media selection, forming an eyecatching communication effect to ensure that the city's image communication keeps pace with the times in the end.

\section{References}

[1] CNNIC's 45th China Internet Statistics Report, https://tech.sina.com.cn/zt_d/cnnic45/:65-69. (in Chinese)

[2] Chen Ying, The Media Construction of City Image Concept Analysis and Theory Frame [J]. Press Circles, 2009 (10): p104. (in Chinese)

[3] He Hui ,Liang Jing, etc. Shaping China's National Image: Forms and Methods [M]. Zhou Mingwei, chief ed. Collection of Researches on National Image Communication [M]. Foreign Languages Press, 2008, p70. (in Chinese)

[4] Liu Jianming, 5G's Historical Disruption of Society and the Media Industry [J]. Journalism Lover, 2019 (3): p7-11. (in Chinese)

[5] Tan Yufei, Beijing City Image Communication: Path Selection in New Media Environment [M]. Beijing: Social Sciences Academic Press, 2019, p30. (in Chinese)

[6] Guo Qingguang. Journalism \& Communication [M]. Beijing: Communication University of China Press, 2011. (in Chinese)

[7] Xu Jian, Shen Jiao. Media identification of city image - 40 years of development of Chinese city image [M]. Shanghai: Shanghai Jiaotong University Press, 2018. (in Chinese) 